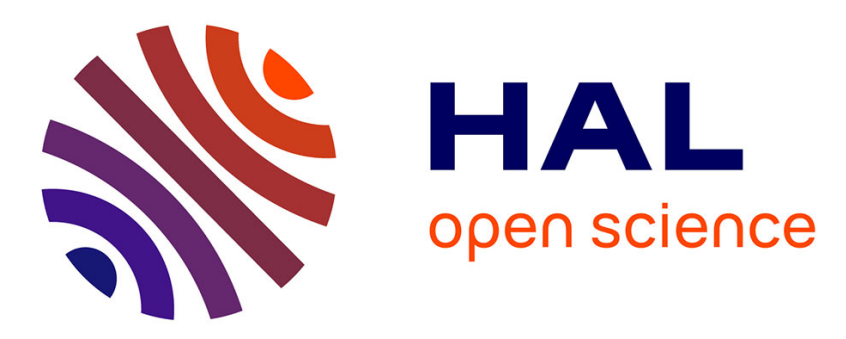

\title{
A Robust Polytopic Approach for State-Dependent Sampling
}

Christophe Fiter, Laurentiu Hetel, Wilfrid Perruquetti, Jean-Pierre Richard

\section{To cite this version:}

Christophe Fiter, Laurentiu Hetel, Wilfrid Perruquetti, Jean-Pierre Richard. A Robust Polytopic Approach for State-Dependent Sampling. ECC 2013: 12th European Control Conference, Jul 2013, Zurich, Switzerland. pp.2603 - 2608. hal-00849833

\section{HAL Id: hal-00849833 \\ https://inria.hal.science/hal-00849833}

Submitted on 7 Sep 2015

HAL is a multi-disciplinary open access archive for the deposit and dissemination of scientific research documents, whether they are published or not. The documents may come from teaching and research institutions in France or abroad, or from public or private research centers.
L'archive ouverte pluridisciplinaire HAL, est destinée au dépôt et à la diffusion de documents scientifiques de niveau recherche, publiés ou non, émanant des établissements d'enseignement et de recherche français ou étrangers, des laboratoires publics ou privés. 


\title{
A Robust Polytopic Approach for State-Dependent Sampling
}

\author{
Christophe Fiter, Laurentiu Hetel, Wilfrid Perruquetti, and Jean-Pierre Richard
}

\begin{abstract}
This work aims at decreasing the number of sampling instants in state feedback control for perturbed linear time invariant systems. The approach is based on linear matrix inequalities obtained thanks to Lyapunov-Razumikhin stability conditions and convexification arguments that guarantee the exponential stability for a chosen decay-rate. First, the method enables to perform a robust stability analysis regarding timevarying sampling and to maximize a lower-bound estimate of the maximal allowable sampling interval, by computing the adequate Lyapunov-Razumikhin function. Then, it makes it possible to design a state-dependent sampling control scheme that enlarges even further the maximal allowable sampling intervals.
\end{abstract}

\section{INTRODUCTION}

Networked Control Systems are often required to share a limited amount of resources, which leads to fluctuations of the sampling interval. From the control theory point of view, these variations bring up new challenges.

In the past decade, several works have concerned the robust stability of sampled-data systems with respect to timevarying sampling ([1], [2], [3], [4], [5], [6], [7], [8], [9], [10], [11]). Intensive research has also been conducted to adapt dynamically the sampling in order to reduce the processor and/or network loads while ensuring the desired control performances. There exist three main approaches:

- Event-triggered control ([12], [13], [14], [15], [16]), in which intelligent sensors send information to the controller when special events occur (i.e. crossing a frontier of the state space). This requires a dedicated hardware.

- Self-triggered control ([17], [18], [19], [20], [21]), which emulates event-triggered control without dedicated hardware, by computing at the sampling instant a lower-bound of the next admissible sampling interval. In these works, the computations for the sampling law are made online.

- State-dependent sampling ([11]): This scheme considers the offline design of a sampling map thanks to a covering of the

This work was supported by Ministry of Higher Education and Research, Nord-Pas de Calais Regional Council and FEDER through the 'Contrat de Projet Etat Region (CPER) 2007-2013'

The research leading to these results has received funding from the European Community's Seventh Framework Programme (FP7/2007-2013) under grant agreement $\mathrm{n}^{\circ}$ 257462: HYCON2 Network of Excellence "HighlyComplex and Networked Control Systems".

This work was supported by the INTERREG IV A 2 Mers Seas Zeen Cross-border Cooperation Program 2007-2013 under SYSIASS 6-20 project.

The authors are with Laboratoire d'Automatique, Génie Informatique et Signal (CNRS UMR 8219), École Centrale de Lille, 59651 Villeneuve d'Ascq, France - W. Perruquetti and J-.P Richard are with the Non-A Project team, INRIA Lille-Nord Europe, France christophe.fiter@centraliens-lille.org, laurentiu.heteldec-lille.fr, wilfrid.perruquettilinria.fr, jean-pierre.richarddec-lille.fr state-space into conic regions, each providing an admissible value of the next sampling instant. This allows to reduce the number of online computations with respect to self-triggered control. Up to now however, only ideal Linear Time-Invariant (LTI) systems have been considered.

In the present work, we consider the case of perturbed LTI systems, with state-bounded unknown exogenous disturbances, and we provide tools for 1) a robust stability analysis regarding time-varying sampling, and 2) a statedependent sampling control. For both applications, we ensure the exponential stability for a given decay-rate, thanks to Lyapunov-Razumikhin stability conditions and convexification arguments. The main contributions are:

- the consideration of an unknown exogenous disturbance with convex embedding techniques in a robust stability analysis regarding time-varying sampling;

- the optimization of the Lyapunov-Razumikhin Function (LRF), taking into account both the effects of the sampling and the perturbation. It optimizes the lower-bound of the sampling map for the state-dependent sampling scheme; - the design of a state-dependent sampling scheme for the case of perturbed LTI systems.

The paper is organized as follows. First, we state the problem in Section II and propose the main stability results in Section III. Then, Sections IV and V provide the stability analysis regarding time-varying sampling and the design of a state-dependent sampling scheme. Finally, simulation results are shown in Section VI before concluding in Section VII. Due to space restrictions, all the proofs are left to the technical report [22].

Notations: $\mathbb{R}_{+}=\{\lambda \in \mathbb{R}, \lambda \geq 0\}, \mathbb{R}^{*}=\{\lambda \in \mathbb{R}, \lambda \neq$ $0\} . \lambda_{\max }(M)$ denotes the largest eigenvalue of a symmetric matrix $M \in \mathbb{R}^{n \times n}$. $\mathbb{S}_{n}^{+}$(resp. $\mathbb{S}_{n}^{+*}$ ) is the set of positive (resp. positive definite) symmetric matrices $P \succeq 0$ (resp. $P \succ 0$ ) in $\mathbb{R}^{n \times n}$. The symmetric elements of a symmetric matrix are denoted by $* .\lfloor x\rfloor$ is the largest integer not greater than $x \in \mathbb{R}$, and $\|$.$\| is the Euclidean norm on \mathbb{R}^{n}$. The cardinal of a finite set $\mathcal{K}$ is denoted $|\mathcal{K}|$.

\section{PROBLEM STATEMENT}

Consider the perturbed LTI system

$$
\dot{x}(t)=A x(t)+B u(t)+E w(t), \quad \forall t \in \mathbb{R}_{+},
$$

where $x: \mathbb{R}_{+} \rightarrow \mathbb{R}^{n}, u: \mathbb{R}_{+} \rightarrow \mathbb{R}^{n_{u}}$, and $w: \mathbb{R}_{+} \rightarrow \mathbb{R}^{n_{w}}$ represent respectively the system state, the control function, and the exogenous disturbances. The matrices $A, B$, and $E$ are constant with appropriate dimensions.

The control is a piecewise-constant state feedback:

$$
u(t)=-K x\left(t_{k}\right), \forall t \in\left[t_{k}, t_{k+1}\right), \forall k \in \mathbb{N},
$$


where $K$ is fixed and such that $A-B K$ is Hurwitz. The sampling instants $t_{k}$ satisfy

$$
t_{k+1}-t_{k}=\tau\left(t_{k}, x\left(t_{k}\right)\right) \equiv \tau_{k} \in\left[\delta, \tau_{\max }\left(x\left(t_{k}\right)\right)\right], \forall k \in \mathbb{N},
$$

with a scalar $\delta>0$ that ensures the well posedness of the system (no Zeno phenomenon issue), a sampling function $\tau: \mathbb{R}_{+} \times \mathbb{R}^{n} \rightarrow \mathbb{R}_{+}$, and a maximal sampling map $\tau_{\max }$ : $\mathbb{R}^{n} \rightarrow \mathbb{R}_{+}$. This sampling map defines the upper-bound of the sampling intervals and can be seen as a maximal timeinvariant sampling function, to be designed.

The exogenous disturbance is assumed to be state-bounded in a similar way as in [17]:

$$
\exists W \geq 0,\|w(t)\|^{2} \leq W\left\|x\left(t_{k}\right)\right\|^{2}, \forall t \in\left[t_{k}, t_{k+1}\right), \forall k \in \mathbb{N} .
$$

Such a perturbation can represent model uncertainties, local nonlinearities, or some kind of measurement noises.

We denote by $\mathcal{S}$ the closed-loop system $\{(1),(2),(3)$, (4) $\}$. For given sampling function $\tau$ and disturbance $w$, the solution of $\mathcal{S}$ with initial value $x_{0}$ is denoted by $x(t)=$ $\varphi_{\tau, w}\left(t, x_{0}\right)$.

In this work, our main objective is to provide a way to enlarge the maximal sampling map $\tau_{\max }$ from (3) while ensuring the exponential stability of the system for a chosen decay-rate $\beta$, also called $\beta$-stability (i.e. such that there exists a scalar $\gamma$ for which all trajectories satisfy $\|x(t)\| \leq$ $\gamma \mathrm{e}^{-\beta t}\left\|x_{0}\right\|$ for any initial condition $x_{0}$ ).

In order to check the $\beta$-stability of $\mathcal{S}$, we use a LyapunovRazumikhin approach ([23]), which is suggested by the delayed nature of the system, since it uses a Zero-Order-Hold control [24]. It allows for deriving stability conditions close to the ones from discrete-time analysis, while guaranteeing a good behaviour of the system between two consecutive sampling instants.

Proposition 1: Consider scalars $\alpha>1, \bar{\sigma}>0,0<\beta \leq$ $\frac{\ln (\alpha)}{2 \bar{\sigma}}$, and $W \geq 0$, and a map $\tau_{\max }: \mathbb{R}^{n} \rightarrow \mathbb{R}_{+}, 0<\delta \leq$ $\tau_{\max }(x) \leq \bar{\sigma}$. If there exists a quadratic function $V(x)=$ $x^{T} P x, P \in \mathbb{S}_{n}^{+*}$ such that

$$
\begin{gathered}
\text { For all } x \in \mathbb{R}^{n}, \text { for all } \sigma \in\left[0, \tau_{\max }(x)\right], \\
\dot{V}\left(\varphi_{\tau_{\max }, w}(\sigma, x)\right)+2 \beta V\left(\varphi_{\tau_{\max }, w}(\sigma, x)\right) \leq 0 \\
\text { whenever } \alpha V\left(\varphi_{\tau_{\max }, w}(\sigma, x)\right) \geq V(x),
\end{gathered}
$$

then the system $\mathcal{S}$ is globally $\beta$-stable.

Note that if $\beta=0$ and the inequality $\dot{V}\left(\varphi_{\tau_{\max }, w}(\sigma, x)\right) \leq$ 0 in $(\mathrm{C} 1)$ is reinforced to be strict, then the LyapunovRazumikhin theory ensures the asymptotic stability.

Throughout this work, we focus on solving two problems. The first one concerns the design of the LRF $V$ :

Problem 1: Find a quadratic LRF $V$ such that there exists a sampling map $\tau_{\max }$ satisfying $(\mathrm{C} 1)$ with a minimum value $\tau^{*}=\inf _{x \in \mathbb{R}^{n}} \tau_{\max }(x)$ as large as possible.

The second problem concerns the design of the sampling map $\tau_{\max }$ :
Problem 2: Given a quadratic LRF $V$, design a lowerbound approximation of the optimal sampling map $\tau_{\text {opt }}^{V}(x)=$ $\max \tau_{\max }(x)$ such that $(\mathrm{C} 1)$ holds.

\section{MAIN STABILITY RESULTS}

In this section, our aim is to derive sufficient stability conditions from Proposition 1 that depend solely on the time variable $\sigma$ and on the sampled-state $x$.

By introducing the dynamics of system $\mathcal{S}$ in (C1) and using some algebraic manipulations, we obtain:

Lemma 2: Consider scalars $\alpha>1, \bar{\sigma}>0,0<\beta \leq \frac{\ln (\alpha)}{2 \bar{\sigma}}$, and $W \geq 0$, and a map $\tau_{\max }: \mathbb{R}^{n} \rightarrow \mathbb{R}_{+}, 0<\delta \leq \tau_{\max }(x) \leq$ $\bar{\sigma}$. If there exist a matrix $P \in \mathbb{S}_{n}^{+*}$ and a scalar $\varepsilon \geq 0$ such that for all $x \in \mathbb{R}^{n}$, and all $\sigma \in\left[0, \tau_{\max }(x)\right]$,

$$
\left[\begin{array}{c}
\Lambda(\sigma) x+J_{w}(\sigma) \\
x \\
w(\sigma)
\end{array}\right]^{T} \Omega\left[\begin{array}{c}
\Lambda(\sigma) x+J_{w}(\sigma) \\
x \\
w(\sigma)
\end{array}\right] \leq 0
$$

with the matrices

$$
\begin{gathered}
\Lambda(\sigma)=I+\int_{0}^{\sigma} \mathrm{e}^{s A} d s(A-B K), \\
J_{w}(\sigma)=\int_{0}^{\sigma} \mathrm{e}^{A(\sigma-s)} E w(s) d s
\end{gathered}
$$

and

$$
\Omega=\left[\begin{array}{ccc}
A^{T} P+P A+\varepsilon \alpha P+2 \beta P & -P B K & P E \\
* & -\varepsilon P & 0 \\
* & * & 0
\end{array}\right],
$$

then the system $\mathcal{S}$ is globally $\beta$-stable.

Note that in (5) appear the sampled state $x\left(t_{k}\right) \equiv x$ and the time $t-t_{k} \equiv \sigma$, but also other terms that result from the unknown exogenous disturbance, $w(\sigma)$ and $J_{w}(\sigma)$. Using equation (4) about the exogenous perturbation, it is possible to remove these unknown terms and to derive sufficient stability conditions in the form of LMIs and of a set of parametric inequalities of the form $x^{T} \Pi(\sigma) x \leq 0$, parametrized by the time $\sigma$.

Theorem 3: Consider scalars $\alpha>1, \bar{\sigma}>0,0<\beta \leq$ $\frac{\ln (\alpha)}{2 \bar{\sigma}}$, and $W \geq 0$, and a map $\tau_{\max }: \mathbb{R}^{n} \rightarrow \mathbb{R}_{+}, 0<\delta \leq$ $\tau_{\max }(x) \leq \bar{\sigma}$. Then, the system $\mathcal{S}$ is globally $\beta$-stable if there exist scalars $\varepsilon \geq 0, \eta \geq 0$, and $\mu \geq 0$, matrices $P, \Psi_{1}$, $\Psi_{2} \in \mathbb{S}_{n}^{+*}$, and $\Psi_{3} \in \mathbb{S}_{n_{w}}^{+*}$, such that

$$
M_{1}+\Psi_{1}+\Psi_{2} \preceq \mu I, \quad\left[\begin{array}{cc}
\Psi_{3}-\eta I & M_{3}^{T} \\
* & -\Psi_{2}
\end{array}\right] \preceq 0,
$$

and

$$
x^{T} \Pi(\sigma) x \leq 0, \forall x \in \mathbb{R}^{n}, \forall \sigma \in\left[0, \tau_{\max }(x)\right],
$$

with

$$
\begin{aligned}
& \Pi(\sigma)=\Lambda(\sigma)^{T} M_{1} \Lambda(\sigma)-\Lambda(\sigma)^{T} P B K-K^{T} B^{T} P \Lambda(\sigma) \\
& \quad-\varepsilon P+M_{2}(\sigma)^{T} \Psi_{1}^{-1} M_{2}(\sigma)+M_{4}(\sigma)^{T} \Psi_{3}^{-1} M_{4}(\sigma) \\
& \quad+W \eta I+\sigma W \mu \lambda_{\max }\left(E^{T} E\right) f_{A}(\sigma) I,
\end{aligned}
$$




$$
\begin{gathered}
M_{1}=A^{T} P+P A+\varepsilon \alpha P+2 \beta P, M_{3}=P E, \\
M_{2}(\sigma)=-P B K+M_{1} \Lambda(\sigma), M_{4}(\sigma)=E^{T} P^{T} \Lambda(\sigma),
\end{gathered}
$$

and

$$
f_{A}(\sigma)=\left\{\begin{array}{c}
\frac{1}{\lambda_{\max }\left(A+A^{T}\right)}\left(\mathrm{e}^{\lambda_{\max }\left(A+A^{T}\right) \sigma}-1\right) \\
\quad \text { if } \lambda_{\max }\left(A+A^{T}\right) \neq 0, \\
\sigma \text { otherwise. }
\end{array}\right.
$$

Remark 1: For any given state $x \in \mathbb{R}^{n}$, the condition (10) remains the same for any state $y=\lambda x, \lambda \in \mathbb{R}^{*}$. Therefore, it is sufficient to work with homogeneous sampling maps of degree 0 (i.e. satisfying $\tau_{\max }(\lambda x)=\tau_{\max }(x)$ for all $x \in \mathbb{R}^{n}$, $\lambda \in \mathbb{R}^{*}$ ) and to check condition (10) over the unit $n$-sphere. This is very similar to the homogeneity properties brought up for the stability conditions in [19] and [11].

Remark 2: In Theorem 3, $P$ corresponds to the LRF matrix, $\varepsilon$ is a tuning parameter introduced by the use of the S-procedure, and the scalars $\eta$ and $\mu$, as well as the matrices $\Psi_{i}$ correspond to degrees of freedom introduced during the majorations of the perturbations $w(\sigma)$ and $J_{w}(\sigma)$ from Lemma 2.

In the next sections, we show how to adapt the obtained stability conditions so as to perform a robust analysis with respect to time-varying sampling, compute all the parameters efficiently, and perform a state-dependent sampling control.

\section{TIME-VARYING SAMPLING AND OPTIMIZATION OF THE PARAMETERS}

In this section, we consider a constant (i.e. stateindependent) sampling map:

$$
\tau_{\max }(x)=\tau_{\max }^{\text {(global) }}, \forall x \in \mathbb{R}^{n},
$$

and look for a solution to Problem 1 by computing:

- a state-independent upper-bound estimation $\tau_{\max }^{\text {(global) }}=\tau^{*}$ for time-varying sampling as in the framework of robust control techniques (i.e. guaranteeing $\beta$-stability for any timevarying sampling bounded by $\left.\tau^{*}\right)$,

- the associated LRF $V(x)=x^{T} P x$ (as well as Theorem 3 parameters $\Psi_{1}, \Psi_{2}, \Psi_{3}, \varepsilon, \eta$ and $\mu$ ).

To this aim, we first rewrite the condition (10) in Theorem 3 as a parameter-dependent LMI as follows.

Lemma 4: The condition (10) in Theorem 3, with the sampling map (14), is satisfied if and only if the parameterdependent LMI

$$
\Delta(\sigma)=\left[\begin{array}{ccc}
R(\sigma) & M_{2}(\sigma)^{T} & M_{4}(\sigma)^{T} \\
* & -\Psi_{1} & 0 \\
* & * & -\Psi_{3}
\end{array}\right] \preceq 0
$$

is satisfied for all $\sigma \in\left[0, \tau_{\max }^{(\text {global })}\right]$, with

$$
\begin{aligned}
R(\sigma)= & \Lambda(\sigma)^{T} M_{1} \Lambda(\sigma)-\Lambda(\sigma)^{T} P B K-K^{T} B^{T} P \Lambda(\sigma) \\
& -\varepsilon P+W \eta I+\sigma W \mu \lambda_{\max }\left(E^{T} E\right) f_{A}(\sigma) I .
\end{aligned}
$$

Then, in order to reduce the number of conditions regarding the time variable to a finite number, we use convex embedding approach:

Convex embedding according to time: The matrix function $\Delta$ is continuous on the compact set $\left[0, \tau_{\max }^{\text {(global) }}\right]$. Therefore, it is possible to design a convex polytope defined by a finite set of vertices $\bar{\Delta}_{\kappa}\left(\tau_{\max }^{\text {(global) }}\right)$, with $\kappa \in \mathcal{K}\left(\tau_{\max }^{\text {(global) }}\right)$ (a finite set of indexes), such that

$$
\begin{gathered}
\left(\bar{\Delta}_{\kappa}\left(\tau_{\max }^{\text {(global })}\right) \preceq 0, \forall \kappa \in \mathcal{K}\left(\tau_{\max }^{(\text {global })}\right)\right) \\
\Downarrow \\
\left(\Delta(\sigma) \preceq 0, \forall \sigma \in\left[0, \tau_{\max }^{(\text {global })}\right]\right) .
\end{gathered}
$$

The form of the matrix function $\Delta$ given by (15) enables to build these vertices as linearly dependent on $P, \Psi_{1}, \Psi_{3}$, $\eta$, and $\mu$. One possible construction of a convex polytope satisfying (17) is proposed in the Appendix, Section VIII. This construction is based on the results from [1], which provide tools to build convex hulls around exponential matrix functions using Taylor polynomials.

With such a convex embedding, and using the results from Lemma 7 and Theorem 3, we obtain the following stability Theorem for systems with time-varying sampling.

Theorem 5: Consider $\varepsilon \geq 0$ a tuning parameter. Let a scalar $0<\tau_{\max }^{\text {(global) }} \leq \bar{\sigma}$ and the constant sampling map defined in (14). Let scalars $\alpha>1, \bar{\sigma}>0,0<\beta \leq \frac{\ln (\alpha)}{2 \bar{\sigma}}$, and $W \geq 0$, and matrices $\bar{\Delta}_{\kappa}\left(\tau_{\max }^{\text {(global) }}\right)$ satisfying (17), with $\kappa \in \mathcal{K}\left(\tau_{\text {max }}^{\text {(global })}\right)$.

If there exist matrices $P, \Psi_{1}, \Psi_{2} \in \mathbb{S}_{n}^{+*}, \Psi_{3} \in \mathbb{S}_{n_{w}}^{+*}$, and scalars $\eta \geq 0$ and $\mu \geq 0$, such that the LMIs (9) and $\bar{\Delta}_{\kappa}\left(\tau_{\max }^{\text {(global })}\right) \preceq 0$ are satisfied for all $\kappa \in \mathcal{K}\left(\tau_{\max }^{\text {(global })}\right)$, then the system (1), subject to perturbations (4), is globally $\beta$ stable with respect to the control (2) for any time-varying sampling bounded by $\tau_{\max }^{\text {(global) }}$.

Based on this result, we provide in the following an algorithm to compute a lower-bound estimate of the maximal allowable sampling interval for time-varying sampling. It is adapted to the polytopic description presented in the Appendix, Section VIII, which is based on Taylor series approximations. These approximations induce an estimation error which can be upper-bounded by a scalar $\nu$.

\section{Algorithm:}

Step 1: First, we consider Theorem 5 and the polytopic description (21) and (22) with $\nu=0$. The search for $P$, $\Psi_{1}, \Psi_{2}, \Psi_{3}, \eta$ and $\mu$ is then an LMI problem, and we may optimize the search of the largest $\tau_{\max }^{\text {(global) }}$ (denoted $\hat{\tau}^{*}$ ) and its associated parameter $\varepsilon$ by using a line-search algorithm. Step 2: Then, we compute the value of the upper-bound $\nu$ corresponding to the obtained $P, \varepsilon, \Psi_{1}, \Psi_{2}, \Psi_{3}, \eta$ and $\mu$. Using this value, it becomes possible to evaluate the matrix inequalities $\bar{\Delta}_{\kappa}\left(\tau_{\max }^{\text {(global) }}\right) \preceq 0$ in Theorem 5 so as to obtain an estimation of the largest upper-bound for time-varying sampling $\tau^{*} \leq \hat{\tau}^{*}$ which satisfies the stability conditions. Step 3: The maximal sampling map is then defined as

$$
\tau_{\max }(x)=\tau^{*}, \forall x \in \mathbb{R}^{n} .
$$




\section{STATE-DEPENDENT SAMPLING}

The state-dependent sampling aims at emulating selftriggered control while trading online computations for offline computations, thus reducing the processor load during the real-time control of the system. In this formulation, the sampling map is defined over regions of the state-space:

$$
\tau_{\max }(x)=\tau_{\max }^{(s)}, \forall x \in \mathcal{R}_{s}, \forall s \in\{1, \cdots, q\} .
$$

Here, the homogeneity brought up in Remark 1 motivates us for working with conic regions of the form

$$
\mathcal{R}_{s}=\left\{x \in \mathbb{R}^{n}, x^{T} \Phi_{s} x \geq 0\right\}, \Phi_{s}=\Phi_{s}^{T} \in \mathbb{R}^{n \times n} .
$$

Possible constructions of such conic regions are presented in [11], using the spherical coordinates of the state or the discrete-time behaviour of the system.

Using the results from Theorem 3 and the convex embedding approach presented in the previous section (with identical notations), we obtain the following stability property for systems with state-dependent sampling:

Theorem 6: Let a matrix $P \in \mathbb{S}_{n}^{+*}$, and scalars $\varepsilon \geq 0$, $\alpha>1, \bar{\sigma}>0,0<\beta \leq \frac{\ln (\alpha)}{2 \bar{\sigma}}$, and $W \geq 0$ be given. Let matrices $\Psi_{1}, \Psi_{2} \in \mathbb{S}_{n}^{+*}, \Psi_{3} \in \mathbb{S}_{n_{w}}^{+*}$, and scalars $\eta \geq 0$ and $\mu \geq 0$, such that the LMIs (9) are satisfied. Consider the sampling map (18) defined on conic regions (19), with sampling intervals $\tau_{\max }^{(1)}, \cdots, \tau_{\max }^{(q)}$ satisfying $0<\delta \leq \tau_{\max }^{(s)} \leq$ $\bar{\sigma}$. Assume there exist matrices $\bar{\Delta}_{\kappa}\left(\tau_{\max }^{(s)}\right)$, with $\kappa \in \mathcal{K}\left(\tau_{\max }^{(s)}\right)$ a finite set, satisfying for all $s \in\{1, \cdots, q\}$, and $\rho_{s} \geq 0$,

$$
\begin{gathered}
\left(\bar{\Delta}_{\kappa}\left(\tau_{\max }^{(s)}\right)+\left[\begin{array}{cc}
\rho_{s} \Phi_{s} & 0 \\
* & 0
\end{array}\right] \preceq 0, \forall \kappa \in \mathcal{K}\left(\tau_{\max }^{(s)}\right)\right) \\
\left(\Delta(\sigma)+\left[\begin{array}{cc}
\rho_{s} \Phi_{s} & 0 \\
* & 0
\end{array}\right] \preceq 0, \forall \sigma \in\left[0, \tau_{\max }^{(s)}\right]\right),
\end{gathered}
$$

with $\Delta(\sigma)$ introduced in (15).

If there exist scalars $\rho_{s} \geq 0$ such that the LMIs $\bar{\Delta}_{\kappa}\left(\tau_{\max }^{(s)}\right)+$ $\left[\begin{array}{cc}\rho_{s} \Phi_{s} & 0 \\ * & 0\end{array}\right] \preceq 0$ are satisfied for all $s \in\{1, \cdots, q\}$ and $\kappa \in \mathcal{K}\left(\tau_{\text {max }}^{(s)}\right)$, then the system $\mathcal{S}$ is globally $\beta$-stable.

Theorem 6 provides sufficient conditions for Theorem 3, and enables to analyse the stability of the system for a given sampling map $\tau_{\max }$ defined on conic regions. Just as in the previous section, the matrices $\bar{\Delta}_{\kappa}\left(\tau_{\max }^{(s)}\right), \kappa \in \mathcal{K}\left(\tau_{\max }^{(s)}\right)$, can be designed following the approach described in the Appendix, Section VIII.

One approach to compute a lower-bound approximation of the optimal sampling map (i.e. a solution of Problem 2), consists in using the LMI conditions from Theorem 6 (with given parameters $P, \varepsilon, \Psi_{1}, \Psi_{2}, \Psi_{3}, \eta, \mu$ and $\nu$ ), in order to maximize the sampling intervals $\tau_{\max }^{(s)}$ on each region with a line search algorithm.

Note that using the LRF $V(x)=x^{T} P x$ and the parameters $\varepsilon, \Psi_{1}, \Psi_{2}, \Psi_{3}, \eta, \mu$ and $\nu$ computed thanks to the algorithm in Section IV allows for designing sampling maps that are lower-bounded by $\tau^{*}$ in the case of state-dependent sampling.

The obtained sampling map (18) can then be used to perform a state-dependent sampling control following the sampling law (3).

\section{NUMERICAL EXAMPLE}

Consider the system from [12]:

$\dot{x}(t)=\left[\begin{array}{cc}0 & 1 \\ -2 & 3\end{array}\right] x(t)-\left[\begin{array}{l}0 \\ 1\end{array}\right]\left[\begin{array}{ll}-1 & 4\end{array}\right] x\left(t_{k}\right)+\left[\begin{array}{ll}1 & 0 \\ 0 & 1\end{array}\right] w(t)$.

We use the polytopic description presented in the Appendix, Section VIII, with a polynomial approximation degree term $N=5$, and $l=100$ polytopic subdivisions.

First, we apply the algorithm proposed in Section IV to perform a robust stability analysis with respect to timevarying sampling for different values of parameters $\beta$ and $W$. The obtained upper-bounds $\tau^{*}$ for time-varying samplings are given in Table I, while Table II presents a comparison with some upper-bounds from the literature, in the unperturbed case, without decay-rate. These upper-bounds are quite close to the practical upper-bound obtained for systems with periodic sampling: $T_{\text {Schur }}=0.5948 \mathrm{~s}$.

\begin{tabular}{|c|c|c|c|}
\hline & $\beta=0$ & $\beta=0.1$ & $\beta=0.3$ \\
\hline$W=0(0 \%)$ & $0.5421 s$ & $0.4404 s$ & $0.3709 s$ \\
\hline$W=0.0025(5 \%)$ & $0.4975 s$ & $0.4092 s$ & $0.2799 s$ \\
\hline$W=0.01(10 \%)$ & $0.4271 s$ & $0.3364 s$ & $0.1573 s$ \\
\hline$W=0.04(20 \%)$ & $0.2719 s$ & $0.1814 s$ & - \\
\hline$W=0.09(30 \%)$ & $0.1417 s$ & $0.0518 s$ & - \\
\hline$W=0.16(40 \%)$ & $0.0322 s$ & - & - \\
\hline
\end{tabular}

TABLE I

MAXIMUM UPPER-BOUNDS $\tau^{*}$ FOR TIME-VARYING SAMPLING

\begin{tabular}{|c|c||c|c|}
\hline Method & $\tau^{*}$ & Method & $\tau^{*}$ \\
\hline$[2]$ & $0.2757 s$ & {$[10]($ LKF approach) } & $0.4305 s$ \\
\hline$[4]$ & $0.3126 s$ & {$[25]$ (Lyapunov + embeddings) } & $0.4578 s$ \\
\hline$[3]$ & $0.3347 s$ & {$[7]$} & $0.5200 s$ \\
\hline$[8]$ & $0.3347 s$ & {$[9]$} & $0.5376 s$ \\
\hline$[5]$ & $0.4244 s$ & Theorem 5 & $0.5421 s$ \\
\hline$[6]$ & $0.4244 s$ & {$[11]($ LRF + embeddings) } & $0.5938 s$ \\
\hline
\end{tabular}

TABLE II

MAXIMUM UPPER-BOUNDS $\tau^{*}$ FOR TIME-VARYING SAMPLING, WITHOUT PERTURBATIONS NOR DECAY-RATE

Second, we set a number $q=100$ of equal conic regions (isotropic partition on the unit sphere $x=\mathrm{e}^{\mathrm{i} \theta}, \theta \in[-\pi, \pi]$, see the design in [11]), and apply the method proposed in Section $\mathrm{V}$, to design the maximal sampling maps for different parameters $\beta$ and $W$. Figure 1 presents the results obtained with $\beta=0.3$. Recall that for each parameter set, $\beta$-stability is ensured for any state-dependent sampling (potentially time-varying) with values under the respective curve in Figure 1 (i.e. satisfying (3)). In the figure, the upperbounds $\tau^{*}$ for time-varying sampling are reminded since they represent a global lower-bound for their respective sampling map. 


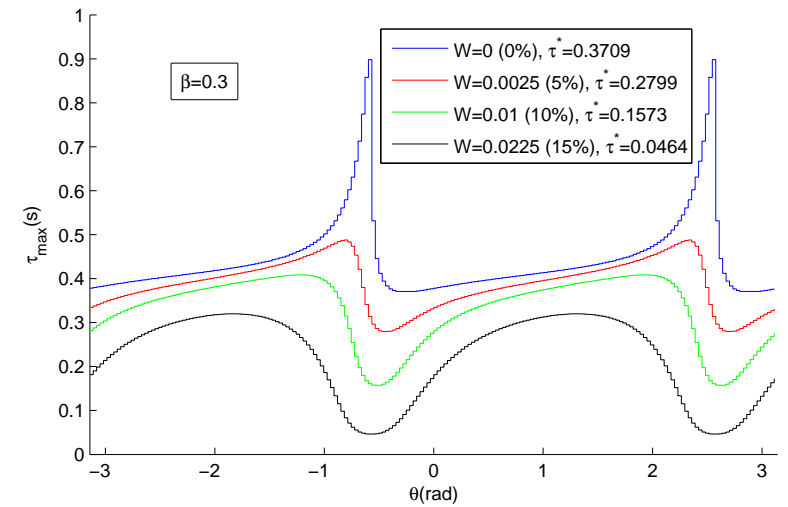

Fig. 1. State-angle dependent sampling map $\tau_{\max }$ for a decay-rate $\beta=0.3$ and different values of $W$

In Figure 2, we present the inter-execution times obtained in a simulation for $\beta=0.3$ and $W=0.01$ (i.e. with perturbations satisfying $\left.\|w(t)\| \leq 10 \%\left\|x\left(t_{k}\right)\right\|\right)$.
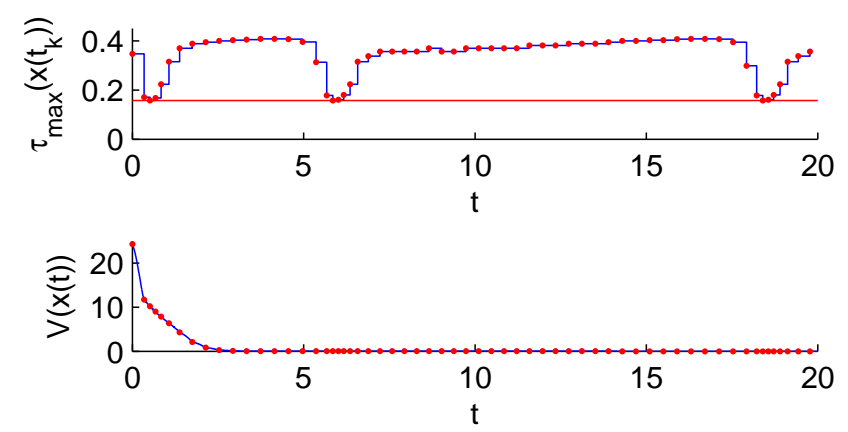

Fig. 2. Inter-execution times $\tau_{\max }\left(x\left(t_{k}\right)\right)$ and LRF $V(x(t))$

\section{CONCLUSION}

We have introduced a design of a maximal state-dependent sampling map $\tau_{\max }$ ensuring the exponential stability with a given decay-rate for perturbed linear state feedback systems. The proposed method, based on convex embeddings and Lyapunov-Razumikhin type stability conditions, can be used to perform both a robust stability analysis with respect to time-varying sampling and a state-dependent sampling control scheme. It presents several advantages:

- It makes it possible to maximize the lower-bound $\tau^{*}$ of the proposed map.

- It provides the corresponding LRF parameters.

- The state-dependent map of the next maximal sampling interval with respect to the past sampled state is designed offline, which helps reducing the real-time processor load.

\section{APPENDIX: POLYTOPIC EMBEDDING DESIGN BASED ON TAYLOR POLYNOMIALS}

We propose a construction of the convex embedding satisfying (17) that is adapted from the results from [1]. Our design is based on a Taylor series approximation of order $N$ performed on $l$ subdivision intervals of $[0, \bar{\sigma}]$.

The objective behind the division of the time interval $[0, \bar{\sigma}]$ into an union of smaller intervals (namely $\left.\bigcup_{j \in\{0, \cdots, l-1\}}\left[j \frac{\bar{\sigma}}{l},(j+1) \frac{\bar{\sigma}}{l}\right]\right)$ is to refine the precision of the convex embedding. It allows for designing small convex polytopes for each time interval subdivision, instead of designing one large one for the whole time interval.

Consider a scalar $0 \leq \sigma^{*} \leq \bar{\sigma}$. In this construction, we define the set of vertex indexes

$$
\mathcal{K}\left(\sigma^{*}\right)=\{0, \cdots, N\} \times\left\{0, \cdots,\left\lfloor\frac{\sigma^{*} l}{\bar{\sigma}}\right\rfloor\right\},
$$

with integers $N \geq 0$ and $l \geq 1$, and design the vertices $\bar{\Delta}_{(i, j)}\left(\sigma^{*}\right)$ for all $(i, j) \in \mathcal{K}\left(\sigma^{*}\right)$, as:

$$
\bar{\Delta}_{(i, j)}\left(\sigma^{*}\right)=\hat{\Delta}_{(i, j)}\left(\sigma^{*}\right)+\nu I
$$

with

$$
\begin{gathered}
\left\{\begin{array}{c}
\hat{\Delta}_{(i, j)}\left(\sigma^{*}\right)=\left(\sum_{k=0}^{i} \tilde{\Delta}_{(k, j)}\left(\frac{\bar{\sigma}}{l}\right)^{k}\right) \text { if } j<\left\lfloor\frac{\sigma^{*} l}{\bar{\sigma}}\right\rfloor, \\
\hat{\Delta}_{(i, j)}\left(\sigma^{*}\right)=\left(\sum_{k=0}^{i} \tilde{\Delta}_{(k, j)}\left(\sigma^{*}-\frac{j \bar{\sigma}}{l}\right)^{k}\right) \text { otherwise, }
\end{array}\right. \\
\tilde{\Delta}_{(0, j)}=\left[\begin{array}{ccc}
L_{0, j} & -K^{T} B^{T} P+\Gamma_{1, j}^{T} M_{1}^{T} & \Gamma_{1, j}^{T} P E \\
* & -\Psi_{1} & 0 \\
* & * & -\Psi_{3}
\end{array}\right], \\
\tilde{\Delta}_{(k \geq 1, j)}=\left[\begin{array}{ccc}
L_{k, j} & \Gamma_{2, j}^{T} \frac{\left(A^{k-1}\right)^{T}}{k !} M_{1}^{T} & \Gamma_{2, j}^{T} \frac{\left(A^{k-1}\right)^{T}}{k !} P E \\
* & 0 & 0 \\
* & * & 0
\end{array}\right], \\
\Gamma_{1, j}=I+N_{j}(A-B K), \Gamma_{2, j}=N_{j}^{\prime}(A-B K),
\end{gathered}
$$

and

$$
\begin{aligned}
L_{0, j}= & \Gamma_{1, j}^{T} M_{1} \Gamma_{1, j}-\varepsilon P+W \eta I \\
& -\Gamma_{1, j}^{T} P B K-K^{T} B^{T} P \Gamma_{1, j}+\tilde{L}_{0, j} \\
L_{1, j}= & \Gamma_{2, j}^{T}\left(M_{1} \Gamma_{1, j}-P B K\right) \\
& +\left(\Gamma_{1, j}^{T} M_{1}^{T}-K^{T} B^{T} P\right) \Gamma_{2, j}+\tilde{L}_{1, j} \\
L_{k \geq 2, j}= & \Gamma_{2, j}^{T} \frac{\left(A^{k-1}\right)^{T}}{k !}\left(M_{1} \Gamma_{1, j}-P B K\right) \\
& +\left(\Gamma_{1, j}^{T} M_{1}^{T}-K^{T} B^{T} P\right) \frac{A^{k-1}}{k !} \Gamma_{2, j} \\
& +\Gamma_{2, j}^{T}\left(\sum_{i=1}^{k-1} \frac{\left(A^{i-1}\right)^{T}}{i !} M_{1} \frac{A^{k-i-1}}{(k-i) !}\right) \Gamma_{2, j}+\tilde{L}_{k, j} .
\end{aligned}
$$

If $\lambda_{\max }\left(A+A^{T}\right)=0$, the matrices $\tilde{L}_{k, j}$ are defined as

$$
\begin{aligned}
& \tilde{L}_{0, j}=W \mu \lambda_{\max }\left(E^{T} E\right)\left(j \frac{\bar{\sigma}}{l}\right)^{2} I, \\
& \tilde{L}_{1, j}=2 W \mu \lambda_{\max }\left(E^{T} E\right) j \frac{\bar{\sigma}}{l} I, \\
& \tilde{L}_{2, j}=W \mu \lambda_{\max }\left(E^{T} E\right) I, \\
& \tilde{L}_{k \geq 3, j}=0 .
\end{aligned}
$$

Otherwise, if $\lambda_{\max }\left(A+A^{T}\right) \neq 0$, they are defined as

$$
\begin{aligned}
\tilde{L}_{0, j}= & W \mu \frac{\lambda_{\max }\left(E^{T} E\right)}{\lambda_{\max }\left(A+A^{T}\right)} j \frac{\bar{\sigma}}{l}\left(\mathrm{e}^{\lambda_{\max }\left(A+A^{T}\right) j \frac{\bar{\sigma}}{l}}-1\right) I, \\
\tilde{L}_{1, j}= & W \mu \frac{\lambda_{\max }\left(E^{T} E\right)}{\lambda_{\max }\left(A+A^{T}\right)} \\
& \left(\mathrm{e}^{\lambda_{\max }\left(A+A^{T}\right) j \bar{\sigma}}\left(1+j \frac{\bar{\sigma}}{l} \lambda_{\max }\left(A+A^{T}\right)\right)-1\right) I, \\
\tilde{L}_{k \geq 2, j}= & W \mu \frac{\lambda_{\max }\left(E^{T} E\right)}{\lambda_{\max }\left(A+A^{T}\right)} \mathrm{e}^{\lambda_{\max }\left(A+A^{T}\right) j \frac{\bar{\sigma}}{l}} \\
& \left(j \frac{\bar{\sigma}\left(\lambda_{\max }\left(A+A^{T}\right)\right)^{k}}{l}+\frac{\left(\lambda_{\max }\left(A+A^{T}\right)\right)^{k-1}}{(k-1) !}\right) I .
\end{aligned}
$$


Finally,

$$
\nu \geq \max _{\substack{\sigma^{\prime} \in[0, \overline{\bar{\sigma}}], r \in\{0, \cdots, l-1\}}} \lambda_{\max }\left(\Delta\left(\sigma^{\prime}+r \frac{\bar{\sigma}}{l}\right)-\sum_{k=0}^{N} \tilde{\Delta}_{(k, r)} \sigma^{\prime k}\right) .
$$

Remark 5: The matrices $\tilde{\Delta}_{(k, j)}$ defined in (24) are the coefficients of the Taylor polynomial of $\Delta$. Indeed, it can be shown (see the proof of Lemma 7 in [22]) that the polynomial approximation of $\Delta$ of order $N$ for $\sigma \in\left[j \frac{\bar{\sigma}}{l},(j+1) \frac{\bar{\sigma}}{l}\right]$, with $j \in\{0, \cdots, l-1\}$, is expressed as $\sum_{k=0}^{N} \tilde{\Delta}_{(k, j)}\left(\sigma-j \frac{\bar{\sigma}}{l}\right)^{k}$. The constant $\nu$ defined in (29) represents an upper-bound of the Taylor series approximation error: it can be shown (see the proof of Lemma 7 in [22]) that $\Delta(\sigma)-\sum_{k=0}^{N} \tilde{\Delta}_{(k, j)}\left(\sigma-j \overline{\frac{\sigma}{l}}\right)^{k} \preceq \nu I$ for all $\sigma \in\left[j \frac{\bar{\sigma}}{l},(j+1) \frac{\bar{\sigma}}{l}\right]$ and $j \in\{0, \cdots, l-1\}$.

Lemma 7: Consider a scalar $0 \leq \sigma^{*} \leq \bar{\sigma}$. The vertices $\bar{\Delta}_{(i, j)}\left(\sigma^{*}\right)$ defined in (22) with the set of indexes $\mathcal{K}\left(\sigma^{*}\right)$ defined in (21) satisfy the property (17): if the condition $\bar{\Delta}_{(i, j)}\left(\sigma^{*}\right) \preceq 0$ is satisfied for all $(i, j) \in \mathcal{K}\left(\sigma^{*}\right)$, then $\Delta(\sigma) \preceq 0$ for all $\sigma \in\left[0, \sigma^{*}\right]$.

\section{REFERENCES}

[1] L. Hetel, J. Daafouz, and C. Iung, "Stabilization of arbitrary switched linear systems with unknown time-varying delays," IEEE Transactions on Automatic Control, vol. 51, no. 10, pp. 1668-1674, 2006.

[2] P. Naghshtabrizi, J.-P. Hespanha, and A.-R. Teel, "Exponential stability of impulsive systems with application to uncertain sampled-data systems," Systems and Control Letters, vol. 57, no. 5, pp. 378-385, 2008.

[3] H. Fujioka, "Stability analysis of systems with aperiodic sample-andhold devices," Automatica, vol. 45, no. 3, pp. 771-775, 2009.

[4] A. Seuret, "Stability analysis for sampled-data systems with a timevarying period," in 48th IEEE Conference on Decision and Control, (Shanghai, China), pp. 8130-8135, 2009.

[5] E. Fridman, "A refined input delay approach to sampled-data control," Automatica, vol. 46, no. 2, pp. 421-427, 2010

[6] K. Liu, V. Suplin, and E. Fridman, "Stability of linear systems with general sawtooth delay," IMA Journal of Mathematical Control and Information, vol. 27, no. 4, pp. 419-436, 2010.

[7] A. Seuret, "A novel stability analysis of linear systems under asynchronous samplings," Automatica, vol. 48, no. 1, pp. 177-182, 2012.

[8] K. Liu and E. Fridman, "Wirtingers inequality and lyapunov-based sampled-data stabilization," Automatica, vol. 48, no. 1, pp. 102-108, 2012.

[9] C. Briat and A. Seuret, "Robust stability of impulsive systems: a functional-based approach," in 4th IFAC conference on Analysis and Design of Hybrid Systems, (Eindhoven, Netherlands), 2012.

[10] C. Fiter, L. Hetel, W. Perruquetti, and J.-P. Richard, "State-dependent sampling for perturbed time-delay systems," in 51st IEEE Conference on Decision and Control, (Maui, Hawaii, USA), 2012.

[11] C. Fiter, L. Hetel, W. Perruquetti, and J.-P. Richard, "A state dependent sampling for linear state feedback," Automatica, vol. 48, no. 8, pp. 1860-1867, 2012.

[12] P. Tabuada, "Event-triggered real-time scheduling of stabilizing control tasks," IEEE Transactions on Automatic Control, vol. 52, no. 9, pp. 1680-1685, 2007.

[13] W. Heemels, J.-H. Sandee, and P. V. Bosch, "Analysis of eventdriven controllers for linear systems," International Journal of Control, vol. 81, no. 4, pp. 571-590, 2008.

[14] J. Lunze and D. Lehmann, "A state-feedback approach to event-based control," Automatica, vol. 46, no. 1, pp. 211-215, 2010.

[15] W. Heemels, M. Donkers, and A. Teel, "Periodic event-triggered control based on state feedback," in 50th IEEE Conference on Decision and Control, (Orlando, Florida, USA), pp. 2571-2576, 2011.
[16] R. Postoyan, A. Anta, D. Nesic, and P. Tabuada, "A unifying lyapunovbased framework for the event-triggered control of nonlinear systems," in 50th IEEE Conference on Decision and Control, (Orlando, Florida, USA), pp. 2559-2564, 2011.

[17] X. Wang and M.-D. Lemmon, "Self-triggered feedback control systems with finite-gain $\mathcal{L}_{2}$ stability," IEEE Transactions on Automatic Control, vol. 54, no. 3, pp. 452-467, 2009.

[18] M. Mazo Jr., A. Anta, and P. Tabuada, "An iss self-triggered implementation of linear controllers," Automatica, vol. 46, no. 8, pp. 1310-1314, 2010.

[19] A. Anta and P. Tabuada, "To sample or not to sample: self-triggered control for nonlinear systems," IEEE Transactions on Automatic Control, vol. 55, no. 9, pp. 2030-2042, 2010.

[20] X. Wang and M.-D. Lemmon, "Self-triggering under state-independent disturbances," IEEE Transactions on Automatic Control, vol. 55, no. 6 , pp. 1494-1500, 2010.

[21] A. Anta and P. Tabuada, "Exploiting isochrony in self-triggered control," IEEE Transactions on Automatic Control, vol. 57, no. 4 , pp. 950-962, 2012.

[22] C. Fiter, L. Hetel, W. Perruquetti, and J.-P. Richard, "Statedependent sampling for perturbed linear time-invariant systems: a polytopic approach," tech. rep., LAGIS, 2013 Available at https://docs.google.com/open?id= 0B0wsY2L71luCRjJyNUVtRXF IR00.

[23] V. Kolmanovskii and A. Myshkis, Applied theory of functional differential equations. Springer, 1992.

[24] E. Fridman, A. Seuret, and J.-P. Richard, "Robust sampled-data stabilization of linear systems: An input delay approach," Automatica, vol. 40, no. 8, pp. 1441-1446, 2004.

[25] C. Fiter, L. Hetel, W. Perruquetti, and J.-P. Richard, "State dependent sampling: an LMI based mapping approach," in 18th IFAC World Congress, (Milan, Italy), 2011. 